\title{
Die Chinesische Medizin - TCM - eine Übersicht
}

\author{
Beginn einer Reihe zur Vorstellung der vier komplementärmedizinischen Fachge- \\ sellschaften, die sich in der UNION zusammengeschlossen haben. Als Erste be- \\ schreibt die «Assoziation Schweizer Ärztegesellschaften für Akupunktur und Chine- \\ sische Medizin» (ASA) ihre häufigsten Therapieverfahren.
}

\section{Albert Naterop-Perroud ${ }^{a}$, Samuel Imfeld ${ }^{b}$}

a Dr. med., Facharzt für Allgemeinmedizin FMH, Inhaber FA Akupunktur-TCM (ASA), Präsident ASA, Vorstandsmitglied UNION

b Dr. med., Facharzt für Allgemeinmedizin FMH Inhaber FA Akupunktur-TCM (ASA), Vorstandsmitglied ASA und UNION
1 Burkhalter D. Bundesrätliche Pressemitteilung vom 12.1.2011.

2 Porkert M. Neues Lehrbuch der chinesischen Diagnostik. Dinkelscherben: Phainon; 1993.

3 Porkert M, Hempen C. Systematische Akupunktur. 2. Auflage. München: Urban \& Schwarzenberg; 1997.

4 Focks C, Hillenbrand N. Leitfaden Traditionelle Chinesische Medizin. 6. Auflage. München: Urban \& Schwarzenberg; 2010.

Korrespondenz:

Dr. med. Albert Naterop-Perroud ASA

Postfach

CH-8575 Bürglen

Tel. 0716300983

naterop-perroud[at]bluewin.ch
In Würdigung der - vorerst provisorischen - Wiederaufnahme von fünf komplementärmedizinischen Behandlungsmethoden in den Leistungskatalog der Grundversicherung [1] entstand der Wunsch, diese von vielen Fachpersonen unverstandenen und deshalb skeptisch bis ablehnend bewerteten Therapieformen in einzelnen Publikationen darzustellen.

Die Chinesische Medizin (aktuelle offizielle Bezeichnung in China) ist das wohl älteste schriftlich dokumentierte Behandlungssystem - einschlägige Unterlagen datieren bis ins 4. Jahrhundert vor unserer Zeitrechnung. Sie ist ein in der traditionellen chinesischen Philosophie (insbesondere Taoismus) fundiertes, durch namhafte Sinologen [2] als wissenschaftlich bezeichnetes medizinisches System. Laut Prof. Manfred Porkert werden lateinische Fachbegriffe der komplexen chinesischen Sprache besser gerecht als die früher verwendete deutsche Nomenklatur, die den Sachverhalt oft ungenügend wiedergeben kann; in der Praxis wird heute die englische Terminologie bevorzugt.

\section{Grundbegriffe}

Das Weltbild, das der Chinesischen Medizin zugrunde liegt, ist dem westlichen naturwissenschaftlich geprägten Menschen primär fremd. Im Gegensatz zur etablierten Naturwissenschaft, die auf einem kausalanalytischen Denkmodell beruht, werden Erkenntnisse mittels einer induktiv-synthetischen Methode gewonnen und Aussagen aufgrund unmittelbarer Beobachtung dynamischer gegenwärtiger Verhältnisse gemacht [3]. Reale Wirkungen in der Gegenwart - also Bewegungen, Funktionen - besitzen keine Quantität (Gewicht, Menge) sondern ausschliesslich Qualität im Sinne einer Richtung, während vergangene Wirkungen nur noch quantitative Aussagen (also fixierte, unbewegte - unbelebte - Masse) zulassen. In diesem Sinne betrachten wir die TCM insgesamt als eine echte Komplementarität zur westlichen, europäisch geprägten Schulmedizin.

Ein energetisches Prinzip, genannt Qi, bringt aufgrund seiner polaren Zustände Yin und Yang in einem dauernden Kreislauf alle Lebensprozesse hervor. Yang beinhaltet den Aspekt der Dynamik, steht für Induk- tion, Aktivität, Veränderung, Zerstreuung, Auflösung und ist deshalb undeterminierbar. Yin bedeutet Struktivität, Materialisierung, Verdichten, Verfestigung, Bewahrung und ist somit determinierbar. Diese beiden Polaritäten bilden keine Gegensätze, sondern gehen untrennbar eine aus der anderen hervor. Der energetische Kreislauf wird bildhaft mit den Jahreszeiten verglichen, denen wiederum fünf Wandlungsphasen (fälschlich «Elemente» genannt) Wasser, Holz, Feuer, Erde und Metall zugeordnet sind. Das Qi zirkuliert in zwölf Funktionskreisen - im weitesten Sinne vergleichbar mit den unsichtbaren Kraftlinien eines Magneten - im Organismus und ist zugänglich für eine Beeinflussung an genau definierten Stellen der Körperoberfläche, den sogenannten Akupunkturpunkten [4].

Wenn das Qi frei und ungehindert im jeweiligen System zirkulieren kann, bedeutet das für einen Organismus Gesundheit, für ein Wirtschaftssystem Prosperität, in einem Staatsgebilde Wohlfahrt.

Durch das Einwirken äusserer Faktoren - äusseren Agenzien - wie Kälte, Hitze, Wind, Feuchtigkeit, Trockenheit; inneren Agenzien (damit sind Emotionen angesprochen) oder durch Fehlverhalten (Diätfehler, Überanstrengung und «Ausschweifungen») wird dieser freie Fluss gestört und Krankheit (oder Wirtschaftskrisen, politische Instabilität) ist die Folge.

Aufgrund einer ausführlichen Anamnese und Inspektion wird eine Arbeitsdiagnose erstellt, die nach den acht Leitkriterien Yin/Yang, oberflächlich/tief, algor/calor (das heisst verminderte/gesteigerte Dynamik) und inanitas/repletio (Mangel bzw. Überfülle) erfolgt. Durch eine minutiöse Pulstastung und die

\section{Steckbrief ASA}

ASA steht für «Assoziation Schweizer Ärztegesellschaften für Akupunktur und Chinesische Medizin». Gegründet wurde sie am 6. Juni 1998. Alle Informationen finden sich auf der Homepage www.akupunktur-tcm.ch

Kontakt:

ASA Sekretariat, Postfach, CH-8575 Bürglen, Tel. 07163009 83, asa[at]akupunktur-tcm.ch 


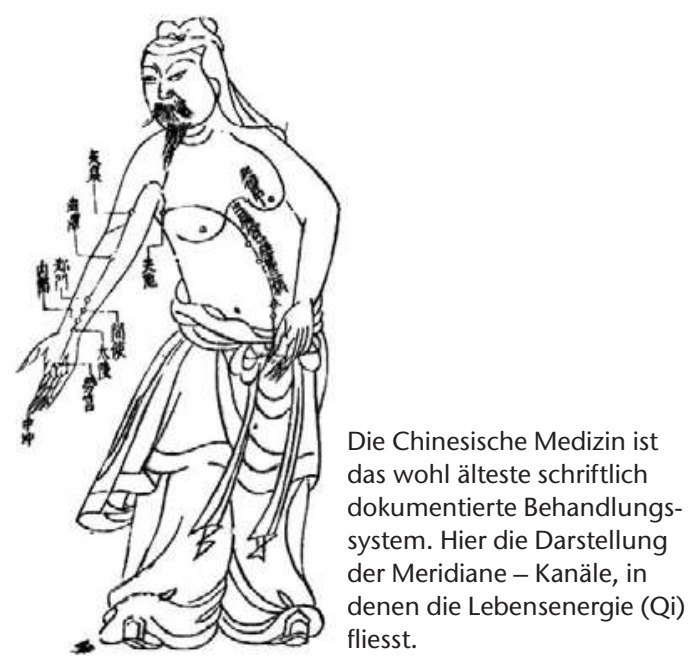

Zungendiagnostik wird die Diagnose verfeinert und bestätigt und daraus folgt unmittelbar die therapeutische Massnahme: Harmonisierung der Dynamik und Korrektur eines Mangels oder einer Überfülle, bezogen auf Yin oder Yang.

\section{Akupunktur und Moxibustion}

In der westlichen Welt sind hauptsächlich die $A k u-$ punktur und die Moxibustion (Stimulation mit glimmendem getrocknetem Beifusskraut) als Behandlungsformen bekannt. Neben segmentalen Wirkungen der Nadel-Behandlung im Zentralnervensystem auf Ebene des Rückenmarkes konnte auch nachgewiesen werden, dass über die Reizung der Haut kutiviszerale Reflexe ausgelöst werden, welche ihrerseits regulierend auf zugehörige Organe einwirken [5]. Die Existenz der sogenannten Head'schen Zonen ist allgemein anerkannt.

Für die klassische chinesische Körper-AkupunkturBehandlung steht ein komplexes System von Energiebahnen, den Leitbahnen oder Meridianen, mit über 300 Akupunktur-Punkten zur Verfügung. Ergänzend werden zahlreiche bewährte Extra- oder ErfahrungsPunkte ausserhalb der Leitbahnen benutzt [5].

In der chinesischen Antike gelangten Knochenoder Stein-Nadeln zur Anwendung. Heute werden an ausgewählten Punkten feine Stahl-Nadeln eingestochen. Sobald die Nadelspitze die korrekte Lage erreicht hat, empfindet der Patient ein kaum beschreibliches dumpfes Gefühl, das sogenannte deqi. Die Nadeln werden stimuliert sowie in der Regel 2030 Minuten im Körper belassen. Je nach Indikation wird während dieser Zeit nachstimuliert, gegebenenfalls zusätzlich mit Moxibustion, und die Prozedur, falls angezeigt, zweimal wöchentlich, in bis zu zwölf, selten auch mehr Sitzungen, wiederholt.

Eine neuere Akupunktur-Form, insbesondere in Europa weitverbreitet, ist die sogenannte Aurikulotherapie, entwickelt Mitte des 20. Jahrhunderts von Dr. Paul Nogier, einem französischen Arzt [6]. Hierbei werden spezielle Nadeln lediglich in die Ohrmuschel gestochen und bei Bedarf auch für einige Tage belas- sen. Die Punktlokalisation geschieht anhand des sogenannten RAC, réflexe-auriculo-cardiaque [6].

Aufgrund zahlloser Erfahrungen von TCM-praktizierenden Kolleginnen und Kollegen und zunehmend belegt durch valide Studienresultate, bewährt sich die Akupunktur insbesondere bei Migräne, Krankheiten des rheumatischen Formenkreises, Dysmenorrhoe und Infertilität sowie Störungen des Verdauungsapparates wie Reizdarmsyndrom, Irritable Bowel Disease und Morbus Crohn.

\section{Diätetik}

Laut traditionellen Texten wird als grundlegendste Massnahme überhaupt die Diätetik in ihrer ureigensten Bedeutung, d.h. die Lebensführung und nicht allein die Ernährungsweise, immer wieder deutlich hervorgehoben [7].

Die Beschäftigung mit Ernährung und Medizin hat in China eine lange Tradition. Bereits im 3. Jahrhundert vor unserer Zeit war man sich der Auswirkungen der Nahrung auf den menschlichen Körper bewusst [7]; allerdings wurde noch keine Unterscheidung zwischen Arzneien und Nahrungsmitteln vorgenommen. Eine klare Differenzierung begann sich erst in der Tang-Dynastie (618-907) abzuzeichnen. Übrigens verschrieben auch die Ärzte des Altertums im Westen häufig Nahrungsmittel als Heilmittel, jedoch wurde die Qualifikation der Wirkungen deutlich weniger kohärent in das medizinische System integriert, und auch heute sind in der westlichen Ernährungslehre erst Ansätze vorhanden, regulative und funktionelle Aspekte zu berücksichtigen; quantitative Faktoren beherrschen die Sichtweise. Die Chinesische Diätetik hingegen beruht nahtlos auf demselben Ordnungsprinzip wie die gesamte Chinesische Medizin. Nahrungsmittel werden aufgrund funktioneller Kriterien eingeteilt: a) nach ihrem Temperaturverhalten, das Auskunft zur energetischen Dynamik gibt, b) nach der Geschmacksrichtung, die Aufschluss bietet über die Schicht (Tiefe), in der sich ihr Effekt entfaltet, c) nach der energetischen Wirktendenz, die angibt, ob eine emporhebende oder absenkende bzw. auf die Oberfläche oder die Tiefe gerichtete Wirkung erzeugt wird, und schliesslich d) anhand ihres Funktionskreisbezuges.

Sun Si Miao schreibt in seinem Standardwerk Qian-ji Yao-fang («Rezepturen, die tausend Goldstücke wert sind», um 650): «Damit der menschliche Körper seine Ausgewogenheit und Harmonie beibehält, ist es einzig erforderlich, sich um eine angemessene Ernährung zu kümmern. Keinesfalls sollte man unkontrolliert Arzneimittel einnehmen, denn ihre Kraft ist einseitig (...) sie führen zu einer Unausgewogenheit des Qi in den Funktionskreisen des Menschen, wodurch [das Eindringen] äusserer Affektionen erleichtert wird. Alle Lebewesen sind abhängig von Nahrung (...) [Die Nahrung] wird von den Leuten zwar täglich verwendet, aber sie wissen so gut wie nichts darüber (...).» 
Wenige Zeilen später schreibt er ins Pflichtenheft der Ärzte: «(...) diejenigen, die Medizin praktizieren, sollten zunächst den Ursprung einer Erkrankung deutlich erkennen; sie sollten wissen, welche Übergriffe [die Krankheit hervorgebracht haben] und sollten sie dann mit diätetischen Methoden behandeln. Erst wenn die Ernährungstherapie keine Heilung bringt, sind Arzneien einzusetzen. Das Wesen der Arzneien ist hart und heftig (...) wird es zu Unglück und Überflutungen kommen, wenn man Arzneimittel [nachlässig] gegen Krankheiten wirft» [7].

Die Diätetik eignet sich also hauptsächlich zur gleichmässigen und sanften Stützung des gesamten menschlichen Organismus. Sie wirkt insbesondere auf die sogenannte «Mitte», Dreh- und Angelpunkt des gesamten Funktionskreissystems und Ort der Entstehung des aktiven energetischen Potentials Qi frumentarium sowie des stofflichen energetischen Potentials Xue (der «Säfte»). Darüber hinaus kann sie auch gezielt auf jeden anderen Funktionskreis und energetische Entgleisungen-gerichtet eingesetzt werden.

\section{Arzneitherapie}

Aus dem bisher Gesagten geht zwanglos die Bedeutung der Arzneitherapie [8] hervor. Die Arzneien werden in derselben Weise wie die Nahrungsmittel eingeteilt; da sie jedoch wesentlich intensivere Wir-
In diesem Zusammenhang muss dringend auf die Gefahr aufmerksam gemacht werden, die durch Selbstmedikation [10] mit über Graumarkt-Kanäle und Internethandel erworbenen Arzneien lauert. Die verbreitete Meinung, es seien ja nur «harmlose Pflanzen», kann bedrohliche Konsequenzen haben. Durch falsche Indikation und unkorrekte Zusammensetzung, nicht deklarierte Zusatzstoffe sowie Pestizide und Schwermetalle sind immer wieder schwerwiegende bis letale Vergiftungen aufgetreten [11] und haben dadurch das Image der Arzneitherapie massgeblich geschädigt.

\section{QiGong, Taiji und Tuina}

QiGong und Taiji sind gymnastische Körperübungen, die den freien Fluss des Qi gezielt harmonisieren und durch eine meditative Ausübung zusätzlich eine entspannende stresslösende Wirkung entfalten. Beide sind nur entfernt mit Krankengymnastik vergleichbar, die einseitig auf mechanische Körperfunktionen ausgerichtet ist.

Das Ziel der Massagetechnik Tuina ist ebenfalls der ungehinderte Fluss des Qi, indem mittels Fingerdruck bewegend und harmonisierend auf die betroffenen Funktionskreise eingewirkt wird. Diese Therapie weist eine gewisse Ähnlichkeit mit manualmedizinischen Massnahmen auf.

\section{«Erkenntnisse werden mittels einer induktiv-synthetischen Methode gewonnen, und Aussagen aufgrund unmittelbarer Beobachtung dynamischer gegenwärtiger Verhältnisse gemacht.»}

8 Hempen $\mathrm{CH}$, Fischer T. Leitfaden Chinesische Phytotherapie. 2. Auflage. München: Urban \& Schwarzenberg; 2007

9 Bühlmann S, Complemedis AG, 4632 Trimbach persönliche Mitteilung.

10 www.theiaforum.org/ Article Folder/herbalmedicines-toxic-effects-druginteractions.pdf

11 www.smw.ch/docs/pdf200x/ 2003/11/smw-10204.PDF

12 www.akupunktur-tcm.ch

13 www.asa-tcm-kongress.ch

14 www.kikom.unibe.ch kungen entfalten, wird zusätzlich auf ihre mögliche Toxizität oder ihre Einsatzmöglichkeit als Antidot hingewiesen. Bei sachgemässer Handhabung können durch ausgeklügelte Vorbehandlungen einerseits sowie geschickte Kombination - zumeist in Form altbewährter klassischer Rezepturen - andererseits, unerwünschte Wirkungen und toxische Risiken wesentlich verringert werden. Bei korrekter Indikation wird auch berichtet, dass der intensive Geschmack der Abkochungen in der Regel von Patienten gut toleriert wird; ein Auftreten von ausgeprägter Aversion spricht für eine falsche oder nicht mehr indizierte Rezeptur. Seit einigen Jahren gelangen neben klassischen Rohdrogen, die einen ziemlich aufwendigen Kochprozess durchlaufen müssen, vermehrt als Granulate und/ oder Tabletten konfektionierte Fertigarzneien bewährter klassischer Rezepte zum Einsatz. Die spezialisierten schweizerischen Apotheken unterziehen übrigens die rezeptierenden Ärzte und Therapeuten einer rigorosen Qualitätskontrolle und weisen unkorrekte Rezepte zurück [9]. Die angebotenen Rohdrogen unterliegen ausserdem schärfsten Qualitätsprozeduren.

\section{Aus-, Weiter- und Fortbildung}

Die «Assoziation der Schweizer Ärztegesellschaften für Akupunktur und Chinesische Medizin» ASA vertritt als Dachorganisation der vier TCM-Ärztegesellschaften über 700 Fachärzte und Fachärztinnen mit einem von der FMH anerkannten Fähigkeitsausweis «Akupunktur - TCM (ASA)». Sie führt modulartige Lehrgänge mit Abschlussprüfungen durch [12]. Jährlich findet der zweitägige ASA TCM-Kongress statt, am 6./7. Dezember 2012 bereits zum sechsten Mal [13].

Die Kollegiale Instanz für Komplementärmedizin KIKOM der Universität Bern [14] ist zuständig für prüfungsrelevante Ausbildungsblöcke auf verschiedenen Stufen des Medizinstudiums.

Wir freuen uns, wenn unsere Ausführungen zur Klärung beitragen und es uns gelingt, eine Brücke schlagen zu helfen zwischen der sogenannten Schulmedizin und Komplementären Heilmethoden. Besonders erfreulich wäre, wenn wir Ansporn geboten hätten, dass Kolleginnen und Kollegen sich für die erweiterten Horizonte der Komplementärmedizin öffnen würden. 